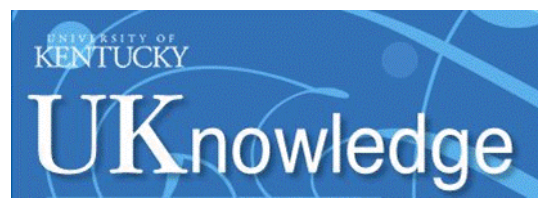

University of Kentucky

UKnowledge

4-15-2015

\title{
The Differences in the Torus Geometry Between Hidden and Non- Hidden Broad Line Active Galactic Nuclei
}

\author{
Kohei Ichikawa \\ Kyoto University, Japan \\ Christopher Packham \\ University of Texas at San Antonio \\ Cristina Ramos Almeida \\ Instituto de Astrofísica de Canarias, Spain \\ Andrés Asensio Ramos \\ Instituto de Astrofísica de Canarias, Spain \\ Almudena Alonso-Herrero \\ California Institute of Technology
}

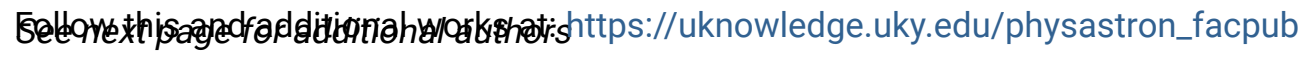

Part of the Astrophysics and Astronomy Commons, and the Physics Commons

Right click to open a feedback form in a new tab to let us know how this document benefits you.

\section{Repository Citation}

Ichikawa, Kohei; Packham, Christopher; Almeida, Cristina Ramos; Ramos, Andrés Asensio; AlonsoHerrero, Almudena; González-Martín, Omaira; Lopez-Rodriguez, Enrique; Ueda, Yoshihiro; Díaz-Santos, Tanio; Elitzur, Moshe; Hönig, Sebastian F.; Imanishi, Masatoshi; Levenson, Nancy A.; Mason, Rachel E.; Perlman, Eric S.; and Alsip, Crystal D., "The Differences in the Torus Geometry Between Hidden and NonHidden Broad Line Active Galactic Nuclei" (2015). Physics and Astronomy Faculty Publications. 249. https://uknowledge.uky.edu/physastron_facpub/249

This Article is brought to you for free and open access by the Physics and Astronomy at UKnowledge. It has been accepted for inclusion in Physics and Astronomy Faculty Publications by an authorized administrator of UKnowledge. For more information, please contact UKnowledge@lsv.uky.edu. 


\section{The Differences in the Torus Geometry Between Hidden and Non-Hidden Broad Line Active Galactic Nuclei}

Digital Object Identifier (DOI)

https://doi.org/10.1088/0004-637X/803/2/57

Notes/Citation Information

Published in The Astrophysical Journal, v. 803, no. 2, 57, p. 1-12.

(C) 2015. The American Astronomical Society. All rights reserved.

The copyright holder has granted the permission for posting the article here.

Authors

Kohei Ichikawa, Christopher Packham, Cristina Ramos Almeida, Andrés Asensio Ramos, Almudena Alonso-Herrero, Omaira González-Martín, Enrique Lopez-Rodriguez, Yoshihiro Ueda, Tanio Díaz-Santos, Moshe Elitzur, Sebastian F. Hönig, Masatoshi Imanishi, Nancy A. Levenson, Rachel E. Mason, Eric S.

Perlman, and Crystal D. Alsip 


\title{
THE DIFFERENCES IN THE TORUS GEOMETRY BETWEEN HIDDEN AND NON-HIDDEN BROAD LINE ACTIVE GALACTIC NUCLEI
}

\author{
Kohei Ichikawa ${ }^{1,14}$, Christopher Packham ${ }^{2}$, Cristina Ramos Almeida ${ }^{3,4,15}$, Andrés Asensio Ramos ${ }^{3,4}$, \\ Almudena Alonso-Herrero ${ }^{5,16}$, Omaira González-Martín ${ }^{3,4}$, Enrique Lopez-Rodriguez ${ }^{2}$, Yoshihiro Ueda ${ }^{1}$, \\ Tanio Díaz-Santos ${ }^{6,7}$, Moshe Elitzur ${ }^{8}$, Sebastian F. Hönig ${ }^{9,15}$, Masatoshi Imanishi ${ }^{10}$, Nancy A. Levenson ${ }^{11}$, \\ Rachel E. Mason ${ }^{12}$, Eric S. Perlman ${ }^{13}$, and Crystal D. Alsip ${ }^{2}$ \\ ${ }^{1}$ Department of Astronomy, Kyoto University, Kitashirakawa-Oiwake-cho, Sakyo-ku, Kyoto 606-8502, Japan; ichikawa@kusastro.kyoto-u.ac.jp \\ ${ }^{2}$ Department of Physics and Astronomy, University of Texas at San Antonio, One UTSA Circle, San Antonio, TX 78249, USA \\ ${ }_{3}$ Instituto de Astrofísica de Canarias, C/Vía Láctea, s/n, E-38205 La Laguna, Tenerife, Spain \\ ${ }_{5}^{4}$ Departamento de Astrofísica, Universidad de La Laguna, E-38205 La Laguna, Tenerife, Spain \\ ${ }^{5}$ Instituto de Física de Cantabria, CSIC-Universidad de Cantabria, E-39005 Santander, Spain \\ ${ }^{6}$ Spitzer Science Center, California Institute of Technology, MS 220-6, Pasadena, CA 91125, USA \\ ${ }^{7}$ Nucleo de Astronomia de la Facultad de Ingenieria, Universidad Diego Portales, Av. Ejercito Libertador 441, Santiago, Chile \\ ${ }^{8}$ Department of Physics and Astronomy, University of Kentucky, Lexington, KY 40506-0055, USA \\ 9 School of Physics and Astronomy, University of Southampton, Southampton SO17 1BJ, UK \\ ${ }^{10}$ Subaru Telescope, 650 North A'ohoku Place, Hilo, HI 96720, USA \\ ${ }^{11}$ Gemini Observatory, Southern Operations Center, c/o AURA, Casilla 603, La Serena, Chile \\ ${ }^{12}$ Gemini Observatory, Northern Operations Center, 670 N. A'ohoku Place, Hilo, HI 96720, USA \\ ${ }^{13}$ Department of Physics and Space Sciences, 150 W. University Blvd., Florida Institute of Technology, Melbourne, FL 32901, USA \\ Received 2014 October 9; accepted 2015 January 26; published 2015 April 15
}

\begin{abstract}
We present results from the fitting of infrared (IR) spectral energy distributions of 21 active galactic nuclei (AGNs) with clumpy torus models. We compiled high spatial resolution $(\sim 0.3-0.7$ arcsec) mid-IR (MIR) $N$-band spectroscopy, $Q$-band imaging, and nuclear near- and MIR photometry from the literature. Combining these nuclear near- and MIR observations, far-IR photometry, and clumpy torus models enables us to put constraints on the torus properties and geometry. We divide the sample into three types according to the broad line region (BLR) properties: type-1s, type-2s with scattered or hidden broad line region (HBLR) previously observed, and type-2s without any published HBLR signature (NHBLR). Comparing the torus model parameters gives us the first quantitative torus geometrical view for each subgroup. We find that NHBLR AGNs have smaller torus opening angles and larger covering factors than HBLR AGNs. This suggests that the chance to observe scattered (polarized) flux from the BLR in NHBLR could be reduced by the dual effects of (a) less scattering medium due to the reduced scattering volume given the small torus opening angle and (b) the increased torus obscuration between the observer and the scattering region. These effects give a reasonable explanation for the lack of observed HBLR in some type-2 AGNs.
\end{abstract}

Key words: galaxies: active - galaxies: nuclei - infrared: galaxies

\section{INTRODUCTION}

While active galactic nuclei (AGNs) present a variety of observational characteristics, the unified model for AGNs proposes the ubiquitous presence of an obscuring torus around their central engines, and that all AGNs are fundamentally the same (Antonucci 1993). This optically and geometrically thick torus produces the effect of a line of sight viewing angle dependency. Type-1 AGNs are observed with a direct view of fast moving material close to the supermassive black hole, resulting in broad emission lines in their spectra, while type-2 AGNs are observed from an edge-on view and the torus blocks the broad emission line region (BLR) component from our line of sight. The most compelling evidence for the unified model was the detection of polarized broad emission lines (PBLs) in type-2 AGNs (e.g., Antonucci \& Miller 1985). Further evidence supporting the unified model comes from infrared (IR) observations of several type-2 AGNs showing the existence of obscured/hidden broad line regions (HBLRs)

\footnotetext{
14 JSPS research fellow (DC1), Japan.

15 Marie Curie fellow.

16 Visiting Professor, Department of Physics and Astronomy, University of Texas at San Antonio.
}

detectable only with dust penetrating IR observations (e.g., Blanco et al. 1990; Nagar et al. 2002; Reunanen et al. 2003; Ramos Almeida et al. 2008).

Against the fact that the observations generally support the unified model, there is the question of why some, but not all, type-2 AGN do not show any observational signs of PBLs. Tran $(2001,2003)$ and Moran et al. (2001) found that only $30 \%-50 \%$ of type-2 AGN show PBLs. Some studies have advocated that the non-detection of a PBL is due to the genuine lack of a BLR (e.g., Tran et al. 2011). Others have suggested that the non-detection is due to obscuration effects, rendering the detection of PBLs difficult or impossible, even with deep near-IR (NIR) spectro-polarimetric observations (Alexander 2001). Using a statistically complete IRAS $60 \mu \mathrm{m}$ selected type-2 AGN catalog, Heisler et al. (1997) investigated the relationship between the detectability of PBLs and IR color as an indicator of the torus inclination angle. They showed that only AGN with a low torus inclination angle have a high detection rate of PBLs compared to those with high inclinations. This result strongly suggests that PBLs could be obscured when there is an edge-on view through the torus and/or nuclear obscuration in the host galaxies. In addition to the optical spectro-polarimetry, X-ray observations suggest that 
there is weak evidence showing different absorptions in two types of type-2 AGN. Gu et al. (2001) found that the AGN with PBL have slightly lower column density $\left(N_{\mathrm{H}}\right)$ than those without PBL. Similarly, Lumsden et al. (2004) and Shu et al. (2007) showed that the detection rate of PBLs decreases as a function of $N_{\mathrm{H}}$, suggesting that the absorption effect by dusty torus could play a role in the detectability of PBL in AGN.

To understand the role of the obscuration by the torus in type-2 AGN and the detectability of PBLs, knowing the torus geometry and properties is crucial. In recent years much progress has been made toward understanding the geometrical structure of the torus. Thanks to the improvement in computing power, more physically realistic torus models assuming "clumpy" distributions (called clumpy torus models) have been coded by several authors (Nenkova et al. 2002, 2008a, 2008b; Hönig et al. 2006; Schartmann et al. 2008; Hönig \& Kishimoto 2010; Stalevski et al. 2012). These models readily reproduce high spatial resolution nuclear NIR to mid-IR (MIR) spectral energy distributions (SEDs) and spectra of AGN with a compact torus of $<10$ pc radius (e.g., Nikutta et al. 2009; Ramos Almeida et al. 2009, 2011a; Alonso-Herrero et al. 2011; Hönig et al. 2011; Lira et al. 2013). On the other hand, traditional smooth torus models (Pier \& Krolik 1992, 1993; Efstathiou \& Rowan-Robinson 1995) had difficulties describing the variety of nuclear SEDs of nearby AGN (e.g., Alonso-Herrero et al. 2003; Gandhi et al. 2009; Asmus et al. 2011; Ichikawa et al. 2012a). Still, the true torus morphology remains far from being conclusively determined until future observations can resolve the torus. Both smooth and clumpy torus models have degeneracies, and from SEDfitting alone it is not possible to choose one or the other (see Feltre et al. 2012 for a comparison between smooth and clumpy torus models).

In this paper, under the assumption that the torus follows a clumpy distribution of dust, which we consider more realistic in principle, we discuss how the precise modeled torus morphology plays a key role in the probability of detecting PBLs by fitting clumpy torus models to our series of IR SEDs. We use 21 high spatial resolution MIR spectra in combination with NIR to far-IR (FIR) photometry, constituting one of the largest compilations of nuclear IR SEDs of AGN in the local universe. These SEDs afforded by $8 \mathrm{~m}$ class telescopes minimize contamination of the MIR torus spectra by surrounding diffuse MIR emission from warm dust and/or star-formation region emission, and hence we are able to construct the highest fidelity torus SED. Therefore we can probe the detectability of the PBL with the least amount of host galaxy contaminations than ever before. The luminosity dependence of the torus morphology will be discussed in a forthcoming paper (K. Ichikawa et al. 2015, in preparation).

\section{OBSERVATIONS}

\subsection{The Sample}

Our principal motivation in this study is to investigate whether the torus model morphology plays a major role in the probability of detecting PBLs. To achieve this goal, we compiled the nearby AGN sources from the MIR samples of González-Martín et al. (2013; 21 sources) and Alonso-Herrero et al. (2011; 13 sources) as both samples already compiled the currently available data set of ground-based $N$ band spectroscopy. Of the 34 sources, five are common to both samples; therefore the total number is 29 sources. We further set the criterion for survey inclusion: the objects must have at least one high spatial resolution NIR $(1-5 \mu \mathrm{m})$ measurement, as the NIR bands significantly help to constrain the torus parameters (Ramos Almeida et al. 2014). Out of 29 sources, 22 fulfilled this criterion. We also removed NGC 1808 from this study as controversy remains to whether it hosts an AGN or ultraluminous X-ray sources in the galactic center due to its low X-ray luminosity $\log L_{2-10 \mathrm{keV}}=40.4 \mathrm{erg} \mathrm{s}^{-1} \quad$ (Scarrott et al. 1993; Jiménez-Bailón et al. 2005). We summarize the properties of the 21 sources in Table 1.

Our sample spans AGN bolometric luminosities taken from the literature $\left(L_{\text {bol }}^{\text {(lit) }}\right)$ in the range $\log L_{\text {bol }}^{\text {(lit) }}=42.7-45.1 \mathrm{erg} \mathrm{s}^{-1}$ (see Table 1), with a mean value of $44.0 \mathrm{erg} \mathrm{s}^{-1}$. This value is fairly consistent with that of magnitude-limited Seyfert catalogs (Maiolino \& Rieke 1995; Ho et al. 1997). This suggests that our sample could be representative of AGNs and their tori in the local universe, although the sample is not complete.

\subsection{New Observations}

We obtained $N$ ( $\mathrm{Si} 2$ filter; the central wavelength with $\lambda_{\mathrm{c}}=8.73 \mu \mathrm{m}$ and $50 \%$ cutoff range of $\left.\Delta \lambda=0.39 \mu \mathrm{m}\right)$ and $Q$ ( $Q a$ filter; $\lambda_{\mathrm{c}}=18.06 \mu \mathrm{m}$ and $\Delta \lambda=0.76 \mu \mathrm{m}$ ) band imaging data of NGC 5135 and NGC 5643, observed by T-ReCS (Program ID GS-2012A-Q-43, PI: Nancy Levenson). The standard MIR chop-nod technique was performed during the observations. The data was reduced using REDCAN(GonzálezMartín et al. 2013).

\subsection{Published Data from the Literature}

We collected the estimated values of the nuclear NIR to MIR emission when available. We compiled nuclear NIR data from both ground- and space-based telescopes such as VLT/NACO and HST/NICMOS. The only exception is the galaxy NGC 5728, whose only NIR flux is from 2MASS (Peng et al. 2006), which we use as an upper limit. Information about the NIR fluxes used here is compiled in Table 2 (columns 2-6).

In order to further reduce the parameter space at longer wavelengths, we used the Spitzer/IRS $30 \mu \mathrm{m}$ continuum fluxes reported by Deo et al. (2009) as upper limits in our fits. We do that because the star formation component can be important at $20-30 \mu \mathrm{m}$, and beyond $30 \mu \mathrm{m}$ completely overwhelms the AGN torus emission in most cases (Netzer et al. 2007) at the spatial resolutions afforded by Spitzer. We only consider the Spitzer $30 \mu \mathrm{m}$ photometry as data points, and also included the Spitzer/IRS spectroscopy when the AGN spectral turnover at $20-30 \mu \mathrm{m}$ is clearly seen in the IRS spectra. This feature suggests that the torus emission is dominant even in the large aperture data from Spitzer (Alonso-Herrero et al. 2012b; Hönig et al. 2014). Only two sources fulfilled the criterion (IC 4329A and MCG 5-23-16). For those galaxies, we also collected available Herschel/PACS data. All the FIR flux information is tabulated in columns 9-11 in Table 2.

The errors were estimated using the prescription given by Alonso-Herrero et al. (2012a). For the NACO AO observations, we used $20 \%$ in $J$ band and $15 \%$ in the HKLM band. For the other ground-based observation data, we applied $30 \%$ for $J$ band, $25 \%$ for $H$ and $K$ bands, and $20 \%$ for $L$ band. These errors include the photometric error, the background subtraction uncertainty, and the uncertainty from estimating the unresolved flux. $M$ band fluxes were always used as upper 
Table 1

Properties of the Sample

\begin{tabular}{|c|c|c|c|c|c|c|c|c|c|c|c|}
\hline $\begin{array}{l}\text { Name } \\
(1)\end{array}$ & $\begin{array}{c}z \\
(2)\end{array}$ & $\begin{array}{c}d \\
(3)\end{array}$ & $\begin{array}{c}\text { Slit/Size } \\
(4)\end{array}$ & $\begin{array}{c}\text { Type } \\
(5)\end{array}$ & $\begin{array}{l}\text { Group } \\
(6)\end{array}$ & $\begin{array}{l}N_{\mathrm{H}} \\
(7)\end{array}$ & $\begin{array}{c}\log L_{\text {bol }}^{\text {(lit) }} \\
(8)\end{array}$ & $\begin{array}{l}b / a \\
(9)\end{array}$ & $\begin{array}{c}A_{\mathrm{V}} \\
(10)\end{array}$ & $\begin{array}{c}i \\
(11)\end{array}$ & $\begin{array}{l}\text { Ref } \\
(12)\end{array}$ \\
\hline NGC 1365 & 0.0055 & 18 & $0.35 / 31$ & Sy 1.8 & Type-1 & 23.6 & 42.9 & 0.5 & $<5$ & $\cdots$ & $(\mathrm{A} 1, \mathrm{~B} 1, \mathrm{~B} 1, \cdots)$ \\
\hline NGC 4151 & 0.0033 & 13 & $0.36 / 23$ & Sy 1.5 & Type-1 & 22.8 & 43.7 & 0.71 & $\cdots$ & $\cdots$ & $(\mathrm{A} 9, \mathrm{~A} 9, \cdots, \cdots)$ \\
\hline NGC 7469 & 0.016 & 66 & $0.75 / 240$ & Sy1 & Type-1 & 20.7 & 45.1 & 0.72 & $\cdots$ & $\cdots$ & $(\mathrm{A} 9, \mathrm{~A} 9, \cdots, \cdots)$ \\
\hline NGC 1068 & 0.0038 & 15 & $0.36 / 26$ & Sy2 & HBLR & $>25$ & 45.0 & 0.85 & $\cdots$ & $60-90$ & $(\mathrm{~A} 2, \mathrm{~A} 9, \cdots, \mathrm{A} 9)$ \\
\hline NGC 3081 & 0.008 & 32 & $0.65 / 100$ & Sy2 & HBLR & 23.9 & 43.8 & 0.8 & $\cdots$ & $\cdots$ & $(\mathrm{A} 3, \mathrm{~B} 2, \cdots, \cdots)$ \\
\hline NGC 3227 & 0.0039 & 17 & $0.75 / 62$ & Sy2 & HBLR & 22.2 & 43.4 & 0.68 & $\cdots$ & $\cdots$ & $(\mathrm{A} 11, \mathrm{~A} 9, \cdots, \cdots)$ \\
\hline Circinus & 0.0014 & 4 & $0.60 / 12$ & Sy2 & HBLR & 24.6 & 43.6 & 0.44 & 9 & $60-90$ & $(\mathrm{~A} 8, \mathrm{~A} 9, \mathrm{~A} 9, \mathrm{~A} 9)$ \\
\hline NGC 5506 & 0.0062 & 25 & $0.36 / 44$ & Sy2 & HBLR & 22.4 & 44.2 & 0.30 & $\geqslant 11$ & 40 & $(\mathrm{~A} 9, \mathrm{~A} 9, \mathrm{~A} 9, \mathrm{~A} 9)$ \\
\hline IC 5063 & 0.011 & 46 & $0.67 / 150$ & Sy2 & HBLR & 23.3 & 44.5 & 0.68 & 7 & $\ldots$ & $(\mathrm{A} 2, \mathrm{~A} 9, \mathrm{~A} 9, \cdots)$ \\
\hline NGC 7582 & 0.0053 & 21 & $0.75 / 76$ & Sy2 & HBLR & 22.7 & 43.3 & 0.42 & 8,13 & $\cdots$ & $(\mathrm{A} 9, \mathrm{~A} 9, \mathrm{~A} 9, \cdots)$ \\
\hline Cen A & 0.0018 & 3 & $0.65 / 11$ & Sy2 & NHBLR & 23.7 & 44.0 & 0.4 & 14.0 & $\cdots$ & $(\mathrm{A} 5, \mathrm{~B} 2, \mathrm{~A} 9, \cdots)$ \\
\hline NGC 5135 & 0.014 & 59 & $0.70 / 200$ & Sy2 & NHBLR & $>25.0$ & 44.4 & 0.7 & $\cdots$ & $\cdots$ & $(\mathrm{A} 2, \mathrm{~B} 2, \cdots, \cdots)$ \\
\hline NGC 5643 & 0.004 & 16 & $0.35 / 29$ & Sy2 & NHBLR & 23.8 & 42.7 & 0.9 & $\cdots$ & $\cdots$ & $(\mathrm{A} 6, \mathrm{~B} 5, \cdots, \cdots)$ \\
\hline NGC 5728 & 0.0094 & 40 & $0.35 / 69$ & Sy2 & NHBLR & 23.6 & 44.5 & 0.6 & $\cdots$ & $\cdots$ & $(\mathrm{A} 7, \mathrm{~B} 6, \cdots, \cdots)$ \\
\hline NGC 7172 & 0.0087 & 35 & $0.36 / 61$ & Sy2 & NHBLR & 22.9 & 43.8 & 0.46 & $\cdots$ & $\cdots$ & $(\mathrm{A} 2, \mathrm{~A} 9, \cdots, \cdots)$ \\
\hline
\end{tabular}

Note. Sample properties. The sample is divided into three subgroups with type-1/HBLR/NHBLR respectively from top to bottom. (1) Object name; (2) redshift; (3) luminosity distance $(\mathrm{Mpc})$ gathered from literature for the case of nearby sources. Within the sample of González-Martín et al. (2013), for NGC 1365, NGC 1386, NGC 1808, NGC 3081, NGC 3281, and Cen A, the values of distance to the galaxies have been taken from Ramos Almeida et al. (2009). For NGC 5643, the distance has been taken from Guainazzi et al. (2004). For the sample of Alonso-Herrero et al. (2011), we gathered them from Alonso-Herrero et al. (2011). For the other sources, we calculated the distances by using cosmological parameter $H_{0}=75 \mathrm{~km} \mathrm{~s}^{-1} \mathrm{Mpc}^{-1}$; (4) slit width (arcsec)/physical size (pc); (5) Seyfert class of AGNs. (6) Sub-group of AGNs. Type-1 represents type-1 AGNs (Sy 1-Sy 1.9) based on optical spectroscopy. HBLR represents type-2 (Sy2) AGNs with hidden broad line region signs, and NHBLR represents type-2 AGNs without any published hidden broad line regions signs. (7) Hydrogen column density; (8) logarithm of bolometric luminosity $\left(\mathrm{erg} \mathrm{s}^{-1}\right.$ ) which is taken from González-Martín et al. (2013) and Alonso-Herrero et al. (2011). We use a typical bolometric correction of 20 (Elvis et al. 1994). (9) The axial ratio; the ratio of the minor to major axis of the host galaxies. All information is taken from González-Martín et al. (2013). (10) Foreground extinction in units of mag; (11) inclination angle of the torus. Levenson et al. (2006) derives the viewing angle of accretion disk of NGC 1386 and we here assume that the accretion disk and the torus are located in the same plane; (12) references of columns (6), (7), (10), and (11). "..." denotes no reference.

References. (A1) Alonso-Herrero et al. (2012a), (A2) Tran (2001), (A3) Moran et al. (2000), (A4) Nicastro et al. (2003), (A5) Alexander et al. (1999), (A6) Gu et al. (2001), (A7) Tran (2003), (A8) Wang \& Zhang (2007), (A9) Alonso-Herrero et al. (2011), (A10) Véron-Cetty \& Véron (2006), (A11) Imanishi (2002), (B1) Tueller et al. (2008), (B2) Marinucci et al. (2012), (B3) Brightman \& Nandra (2011), (B4) Itoh et al. (2008), (B5) Guainazzi et al. (2004), (B6) Goulding et al. (2012), (C1) Levenson et al. (2006)

limits due to the difficulties of estimating the unresolved component. For the NICMOS observations, we used $20 \%$ for the $J$ band, and $20 \%$ for the $H$ and $K$ bands. For the $N$ and $Q$ bands, we use $15 \%$ and $25 \%$ errors, respectively.

\subsection{Subsample}

To examine the torus model properties of different AGN populations, we divide the sample into subgroups based on whether or not the source has signs of HBLR in previously published observations. We first divide the sample into type-1 and type-2 AGNs. Although Seyfert 1.8/1.9 are very ambiguous objects (e.g., see Elitzur et al. 2014 for details), here we define type-1 as AGNs that have at least one broad emission line in their optical spectra. Therefore, we consider Seyfert 1 to 1.9 as type- 1 AGNs and Seyfert 2 as type- 2 AGNs. Next, we divide the type-2 AGNs into those with published polarized BLR detections in the optical and/or in the NIR (HBLR) and those without (non-HBLR, hereafter NHBLR). We use Marin (2014), who compiled almost all of the previously published polarization information of nearby AGNs. These spectro- polarimetric data are taken from several large surveys including the IR-selected sample of Heisler et al. (1997), the FIR fluxlimited sample of Lumsden et al. (2001), the distance-limited sample of Moran et al. (2000, 2002), and the heterogeneous optical- and MIR-selected sample of Tran (2001, 2003). The spectro-polarimetric observations were mainly conducted with small or medium size telescopes (up to $4 \mathrm{~m}$ class), while only NGC 3081 has been confirmed to have HBLR features with the Keck 10 m telescope (Moran et al. 2000). Therefore, we should note that some HBLR AGNs could contaminate the subgroup of NHBLRs in cases where the BLR is below the signal-tonoise afforded by the $4 \mathrm{~m}$ class telescope observations (see C. Ramos Almeida et al. 2015, in preparation). Some sources have currently no published spectro-polarimetric data, but have clear broad emission lines in NIR wavelengths. These sources are MCG 5-23-16, NGC 2110, and NGC 7582 (AlonsoHerrero et al. 2011). All the references used for dividing the sample into each subgroup are indicated in column 12 in Table 1.

Finally, the sources in this study are categorized into three groups (type-1, HBLR, and NHBLR; see column 6 in Table 1). 
Table 2

List of Photometry

\begin{tabular}{|c|c|c|c|c|c|c|c|c|c|c|c|}
\hline $\begin{array}{l}\text { Name } \\
(1)\end{array}$ & $\begin{array}{c}J \\
(2)\end{array}$ & $\begin{array}{c}H \\
(3)\end{array}$ & $\begin{array}{c}K \\
(4)\end{array}$ & $\begin{array}{c}L \\
(5)\end{array}$ & $\begin{array}{l}M \\
(6)\end{array}$ & $\begin{array}{l}N \\
(7)\end{array}$ & $\begin{array}{c}Q \\
(8)\end{array}$ & $\begin{array}{c}30 \mu \mathrm{m} \\
(9)\end{array}$ & $\begin{array}{c}70 \mu \mathrm{m} \\
(10)\end{array}$ & $\begin{array}{c}160 \mu \mathrm{m} \\
(11)\end{array}$ & $\begin{array}{l}\text { Ref } \\
(12)\end{array}$ \\
\hline \multicolumn{12}{|c|}{ Type-1 } \\
\hline NGC 1365 & $\cdots$ & $8.3 \pm 0.83$ & $\ldots$ & $\ldots$ & $\ldots$ & $203 \pm 30.0$ & $818 \pm 204$ & $<12.3$ & $\cdots$ & $\ldots$ & $(\mathrm{A} 1, \mathrm{~B} 1)$ \\
\hline NGC 4151 & $69 \pm 14$ & $104 \pm 10.4$ & $178 \pm 17.8$ & $<325$ & $\cdots$ & $1320 \pm 198$ & $3200 \pm 800$ & $<3.64$ & $\cdots$ & $\cdots$ & $(\mathrm{A} 7, \mathrm{~A} 7)$ \\
\hline IC 4329 A & $\ldots$ & $50.0 \pm 8.0$ & $102 \pm 10$ & $<210$ & $\ldots$ & $1014 \pm 150$ & $\ldots$ & $1.52 \pm 0.015$ & $<1.79$ & $<0.97$ & $(\mathrm{~A} 7, \mathrm{~A} 7)$ \\
\hline NGC 7469 & $16 \pm 3.2$ & $40 \pm 4.0$ & $68 \pm 6.8$ & $<84$ & $\cdots$ & $506 \pm 76$ & $1350 \pm 340$ & $\cdots$ & $\cdots$ & $\cdots$ & $(\mathrm{A} 7, \mathrm{~A} 7)$ \\
\hline \multicolumn{12}{|c|}{ HBLR } \\
\hline NGC 1068 & $9.8 \pm 2.0$ & $98.0 \pm 15.0$ & $445 \pm 100$ & $920 \pm 140$ & $2270 \pm 340$ & $10000 \pm 1500$ & $21800 \pm 5400$ & $\cdots$ & $\cdots$ & $\cdots$ & $(\mathrm{A} 7, \mathrm{~A} 7)$ \\
\hline NGC 2110 & $\cdots$ & $\cdots$ & $\cdots$ & $<33.0$ & $<198$ & $294 \pm 44$ & $561 \pm 140$ & $<0.8$ & $\cdots$ & $\cdots$ & $(\mathrm{A} 7, \mathrm{~A} 7)$ \\
\hline MCG 5-23-16 & $1.1 \pm 0.33$ & $3.7 \pm 0.93$ & $10.7 \pm 2.7$ & $79.5 \pm 16.0$ & $<139.4$ & $633 \pm 95$ & $1450 \pm 360$ & $\ldots$ & $<1.45$ & $<0.45$ & (A7,A7) \\
\hline NGC 3081 & $\ldots$ & $0.22 \pm 0.04$ & $\cdots$ & $\ldots$ & $\ldots$ & $83 \pm 12.5$ & $231 \pm 57.8$ & $<1.10$ & $\ldots$ & $\ldots$ & $(\mathrm{A} 2, \mathrm{~B} 1)$ \\
\hline NGC 3227 & $\cdots$ & $11 \pm 1.1$ & $23 \pm 2.3$ & $<47.0$ & $\ldots$ & $320 \pm 48$ & $1100 \pm 275$ & $<1.76$ & $\cdots$ & $\cdots$ & $(\mathrm{A} 7, \mathrm{~A} 7)$ \\
\hline Circinus & $<1.60$ & $4.77 \pm 0.72$ & $19 \pm 2.9$ & $380 \pm 57$ & $1900 \pm 285$ & $5600 \pm 840$ & $12800 \pm 3200$ & $\ldots$ & $\ldots$ & $\ldots$ & $(\mathrm{A} 7, \mathrm{~A} 7)$ \\
\hline NGC 5506 & $13 \pm 3.0$ & $53 \pm 8.0$ & $80 \pm 12$ & $290 \pm 44$ & $<530$ & $900 \pm 135$ & $2200 \pm 550$ & $<4.05$ & $\ldots$ & $\ldots$ & $(\mathrm{A} 7, \mathrm{~A} 7)$ \\
\hline IC 5063 & $\ldots$ & $0.3 \pm 0.1$ & $4.8 \pm 1.0$ & $\ldots$ & $\ldots$ & $925 \pm 139$ & $\ldots$ & $<3.89$ & $\ldots$ & $\ldots$ & (A7,A7) \\
\hline NGC 7582 & $\cdots$ & $11.0 \pm 1.6$ & $18.0 \pm 2.7$ & $96.0 \pm 14.4$ & $141 \pm 21$ & $384 \pm 57$ & $527 \pm 132$ & $\cdots$ & $\cdots$ & $\cdots$ & $(\mathrm{A} 7, \mathrm{~A} 7)$ \\
\hline NGC 7674 & $1.25 \pm 0.25$ & $5.0 \pm 0.5$ & $12.3 \pm 3.1$ & $53.0 \pm 11.0$ & $<108$ & $518 \pm 78$ & $\cdots$ & $<1.83$ & $\cdots$ & $\cdots$ & $(\mathrm{A} 7, \mathrm{~A} 7)$ \\
\hline \multicolumn{12}{|c|}{ NHBLR } \\
\hline NGC 1386 & $\ldots$ & $0.2 \pm 0.04$ & $\ldots$ & $\ldots$ & $\ldots$ & $147 \pm 22.1$ & $457 \pm 114$ & $<1.58$ & $\ldots$ & $\ldots$ & $(\mathrm{A} 2, \mathrm{~B} 1)$ \\
\hline NGC 3281 & $\ldots$ & $1.3 \pm 0.33$ & $7.7 \pm 1.93$ & $103 \pm 20.6$ & $<207$ & $355 \pm 53.3$ & $1110 \pm 278$ & $\ldots$ & $\ldots$ & $\ldots$ & $(\mathrm{A} 4, \mathrm{~B} 1)$ \\
\hline Cen A & $1.3 \pm 0.26$ & $4.5 \pm 0.68$ & $34 \pm 5.1$ & $200 \pm 30$ & $\ldots$ & $710 \pm 107$ & $2630 \pm 658$ & $\ldots$ & $\ldots$ & $\ldots$ & $(\mathrm{A} 5, \mathrm{~B} 1)$ \\
\hline NGC 5135 & $<0.72$ & $0.66 \pm 0.07$ & $\ldots$ & $\ldots$ & $\ldots$ & $56.36 \pm 8.454$ & $218.92 \pm 54.73$ & $<3.03$ & $\ldots$ & $\cdots$ & (A2;A6, B2) \\
\hline NGC 5643 & $\ldots$ & $<1.7$ & $\ldots$ & $\ldots$ & $\ldots$ & $101.31 \pm 15.17$ & $883.2 \pm 220.8$ & $\ldots$ & $\ldots$ & $\cdots$ & $(\mathrm{A} 2, \mathrm{~B} 2)$ \\
\hline NGC 5728 & $\ldots$ & $\ldots$ & $<7.1$ & $\ldots$ & $\ldots$ & $25 \pm 3.75$ & $184 \pm 46$ & $\ldots$ & $\ldots$ & $\ldots$ & $(\mathrm{A} 6, \mathrm{~B} 1)$ \\
\hline NGC 7172 & $\ldots$ & $<0.4$ & $3.4 \pm 0.86$ & $30 \pm 6$ & $<61.4$ & $165 \pm 30$ & $\ldots$ & $<0.98$ & $\ldots$ & $\ldots$ & $(\mathrm{A} 7, \mathrm{~A} 7)$ \\
\hline
\end{tabular}

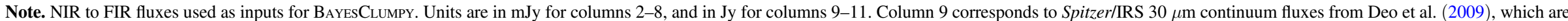

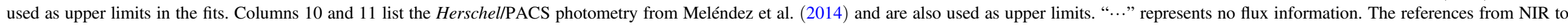
MIR band fluxes are tabulated at column 12 .

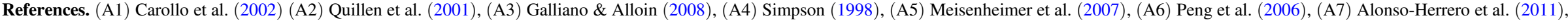

(B1) Ramos Almeida et al. (2009), (B2) This work. 
Table 3

Free Parameters of the BAYESClumpy

\begin{tabular}{lc}
\hline \hline Parameters & Parameter Range \\
\hline Torus radial thickness $(Y)$ & {$[5,30]$} \\
Torus angular width $(\sigma)$ & {$\left[15^{\circ}, 70^{\circ}\right]$} \\
Number of clouds along an equatorial ray $\left(N_{0}\right)$ & {$[1,15]$} \\
Index of the radial density profile $(q)$ & {$[0,3]$} \\
Viewing angle $(i)$ & {$\left[0^{\circ}, 90^{\circ}\right]$} \\
Optical depth of each cloud $\left(\tau_{V}\right)$ & {$[5,150]$}
\end{tabular}

Note. Torus radial thickness $Y$ is defined as $Y=r_{\text {out }} / r_{\text {in }}$, where $r_{\text {out }}$ is the outer radius and $r_{\text {in }}$ is the inner radius. The cloud distribution between $r_{\text {out }}$ and $r_{\text {in }}$ is parameterized as $r^{-q}$.

The sample contains four type-1, 10 HBLR, and seven NHBLR AGNs.

\section{APPLICATION OF TORUS MODEL}

\subsection{Clumpy Torus Model}

We fit the clumpy torus models of Nenkova et al. (2008a), known as $C L U M P Y$, to the data using a Bayesian approach (BAyesClumpy; Asensio Ramos \& Ramos Almeida 2009). Here we describe the six free CLUMPY model parameters used for the SED fitting and the model set up; they are listed in Table 3. The torus clumps are distributed in a smooth, rather than sharp, toroidal-shaped boundary of angular width $\sigma$. The inner radius $\left(r_{\text {in }}\right)$ of the torus is set by the location of the dust at the sublimation temperature $\left(T_{\text {sub }} \sim 1500 \mathrm{~K}\right)$. This is computed using the AGN bolometric luminosity $L_{\text {bol }}(\mathrm{AGN})$

$$
r_{\text {in }}=0.4\left(\frac{L_{\text {bol }}(\mathrm{AGN})}{10^{45} \mathrm{erg} \mathrm{s}^{-1}}\right)^{0.5} \mathrm{pc} .
$$

The torus has a radial extent $(Y)$ defined by $Y=r_{\text {out }} / r_{\text {in }}$, where $r_{\text {out }}$ is the outer radius of the torus. The average number of clouds along the line of sight $\left(N_{\mathrm{LOS}}\right)$ at a viewing angle $i$ is set as

$$
N_{\mathrm{LOS}}=N_{0} \exp \left[-\frac{(90-i)^{2}}{\sigma^{2}}\right],
$$

where $N_{0}$ is the average number of clouds along the radial equatorial ray. $N_{\text {LOS }}$ allows us to derive the escape probability of photons from the AGN central engines $\left(P_{\mathrm{esc}}\right)$. In the CLUMPY dust distribution, the classification of type-1 or type2 AGN depends on whether or not there is a clump along the line of sight, which is a function of the viewing angle of the torus, the number of clumps, and the torus width. This is different from smooth torus models, for which the classification of an AGN as either type- 1 or type- 2 is solely determined by the viewing angle. The escape probability of photons passing through the torus at a given viewing angle $(i)$ can be calculated as

$$
P_{\mathrm{esc}} \sim e^{-N_{\mathrm{LOS}}}
$$

In the CLUMPY model, the radiative transfer equations are solved for each clump and thus the calculations depend on the clump distribution within the torus, the optical depth of each clump, and also its dust composition. Here we assume each clump has the same optical depth $\left(\tau_{V}\right)$, which is defined at the optical $V$ band. The CLUMPY model applies a standard cold oxygen-rich interstellar medium dust, called OHMc dust (Ossenkopf et al. 1992). The torus clumps are distributed as a power law with index $q$ as a function of radius, $N(r) \propto r^{-q}$.

In addition to these six physical parameters, we add two additional parameters to be fitted or fixed. The first parameter is the foreground extinction $\left(A_{V}\right)$, unrelated to the torus. Some authors demonstrated that some AGNs have an extremely deep $9.7 \mu \mathrm{m}$ silicate absorption feature that cannot be reproduced solely by the torus obscuration (Alonso-Herrero et al. 2003, 2011; Polletta et al. 2008; Goulding et al. 2012; GonzálezMartín et al. 2013). They suggested that dust in inclined host galaxies can contribute significantly to the observed SED and silicate feature absorption. Out of the 21 sources 10 are inclined galaxies with low minor-to-major axis ratios $(b / a \leqslant 0.5$; see Table 1). Therefore, some portion of the observed SED is accounted for by the cool foreground dust extinction. AlonsoHerrero et al. (2011) discussed this issue and concluded that for $A_{V} \geqslant 5$, the effects of foreground extinction cannot be ignored when reproducing the silicate $9.7 \mu \mathrm{m}$ feature. We gathered available values of foreground $A_{V}$ from the literature and compiled them in column 10 in Table 1 . The other additional parameter accounts for the multiplicative factor that has to be applied to match the fluxes of a given model to an observed SED. Deriving this factor enables us to calculate the model AGN bolometric luminosity $L_{\text {bol }}^{(\bmod )}$ (Nenkova et al. 2008b). As shown by Alonso-Herrero et al. (2011), $L_{\text {bol }}^{(\bmod )}$ reproduces well the values of $L_{\mathrm{bol}}^{\text {(lit) }}$ for Seyfert galaxies, and therefore, in the following we will refer to $L_{\text {bol }}^{(\bmod )}$ as the bolometric luminosities of the sample studied here.

By combining the derived output parameters of the CLUMPY model, we can derive other important torus morphological parameters as the torus outer radius $r_{\text {out }}$, defined as:

$$
r_{\text {out }}=r_{\text {in }} Y \text { pc. }
$$

We can also calculate the torus scale height $H$ as:

$$
H=r_{\text {out }} \sin \sigma \text { pc. }
$$

Finally, we define the "geometrical" torus covering factor, which is unaffected by the viewing angle, and it is defined by integrating the AGN escape probability over all angles (Nenkova et al. 2008a). This can be written as

$$
C_{\mathrm{T}}=1-\int_{0}^{\pi / 2} P_{\mathrm{esc}}(\beta) \cos (\beta) d \beta,
$$

where $\beta=\pi / 2-i$. Considering that our motivation is to characterize the intrinsic torus morphology, the "geometrical" torus covering factor is more relevant here than the apparent covering factor.

\subsection{BAYESCLUMPY and Modeling Details}

The CLUMPY database currently contains more than $5 \times 10^{6}$ models. Therefore, when fitting the models to the observations, inherent degeneracies have to be taken into account. We then use the BAYEsClumpy fitting tool (Asensio Ramos \& Ramos Almeida 2009), as it performs a fast synthesis of the CLUMPY SEDs. In the last version of BAYESCLUMPY the inference over the model parameters can be done either using neural network interpolation or multilinear interpolation in the full database. After running several tests, Ramos Almeida et al. (2014) concluded that the latter interpolation produces more 
robust results. Therefore, here we use linear interpolation, which results in slight differences in the fitted parameters (within $1 \sigma$ for the majority of the fits) for the 13 galaxies that were modeled by Alonso-Herrero et al. (2011) using the neural network interpolation and subsequently re-fitted in this paper.

BAYESCLUMPY can be used to fit photometry and/or spectra in a Bayesian scheme, carrying out inference over the model parameters for observed SEDs. This way we can specify a priori information about the model parameters. Here we consider uniform prior distribution in the range of each parameter, as summarized in Table 3. The prior distribution of inclination angle $(i)$ is fixed from previous observations if available in the literature, following the same approach as in Alonso-Herrero et al. (2011). From the objects in our sample taken from González-Martín et al. (2013), NGC 1386 has two possible inclination angles, $65^{\circ}$ and $85^{\circ}$ (Levenson et al. 2006). Thus, we use a uniform prior in the range $60^{\circ}-90^{\circ}$ for this source. For the galaxies taken from Alonso-Herrero et al. (2011), we use the same inclination angle constraints they employed, which are compiled in column 11 in Table 1.

We finally include the direct emission of AGN (i.e., a broken power law) which is defined in Equation (13) of Nenkova et al. (2008a) in the SED for type-1 AGNs, in order to reproduce the flatter slope of the NIR band (see Ramos Almeida et al. 2014 for further details).

\section{RESULTS AND DISCUSSIONS}

\subsection{IR SEDs with BAYESCLUMPY Fitting}

The results of the fitting process to the IR SEDs are the posterior distributions for the six parameters that describe the model (defined in Table 3 ), the foreground extinction, and the multiplicative factor needed to match the SED fluxes. However, we can also translate the results into corresponding spectra, as shown in Figure 1.

Figure 1 shows the observed SEDs and nuclear MIR spectra (black filled dots) of the galaxies with the best fit results overlaid (blue solid lines), based on the inference done with BAyESClumpy. The fitted models correspond to those described by the median of the posterior distribution of each parameter. All the derived torus parameters obtained from BAyesClumpy are presented in Table 4.

Some SEDs show lower $Q$-band fluxes than those predicted by the fitted model. This effect is prominent when the silicate $9.7 \mu \mathrm{m}$ feature is observed in deep absorption. Although this may suggest that the model spectra still have difficulty in reproducing the $18 \mu \mathrm{m}$ silicate feature, the difference is only within a factor of 3 in the worst case (NGC 3281). See Ramos Almeida et al. (2009) for further discussion on the $Q$-band excess.

The SEDs of NGC 5506 and NGC 7172 show NIR excesses compared to the model spectra as shown in Figure 1. NIR interferometric observations (Kishimoto et al. 2009, 2011) and NIR reverberation mapping (Kishimoto et al. 2007; Koshida et al. 2009) of Seyfert galaxies suggest that the AGN torus has much smaller sublimation radius than expected from Equation (1). Kawaguchi \& Mori (2011) also showed in their model that if they add the rim darkening effect of the accretion disk, the inner radius of the torus naturally connects to the outer disk. Thus, the NIR excess is readily accounted for by this connection. This is also shown in Stalevski et al. (2012) when they apply the rim darkening effect to produce the model SEDs.
However, in this study, we neither included the SED of a hot dust component nor applied such torus geometry including rim darkening effect for the fitting as this hot dust remains rather unconstrained. Further discussion on the possible origins of NIR excesses can be found in Alonso-Herrero et al. (2011).

\subsection{General Torus Properties for Whole Sample}

In this section, we describe how we obtained the global distribution of the torus model parameters for the whole sample and the three subgroups considered here. To take full advantage of the data employed here, we apply a hierarchical Bayesian approach to derive information about the global distribution of the $C L U M P Y$ parameters for a given subgroup. We use a generalized beta distribution as the prior for each parameter (given that they are defined in closed intervals) and learn the hyperparameters of the prior using importance sampling (e.g., Brewer \& Elliott 2014). This allows us to derive the posterior distribution for each parameter taking into account all the observed data that belongs to an AGN subgroup.

In Table 5 we report the median parameters of the global posterior distributions for the whole sample, as well as for each subgroup. Note that the global posterior distribution of each parameter can be derived only in the circumstance where the prior distribution is the same for all the sources. This is not the case for the inclination angle because we use the constrained prior distributions for some sources as described in Section 3. For the inclination angle, we derive the median parameters of the individual galaxy fits within the each subgroup.

Based on interferometric observations, Kishimoto et al. (2011) reported a typical torus half-light radius of $\sim 1 \mathrm{pc}$ for local AGNs at MIR wavelengths. Our derived torus outer radius $r_{\text {out }}=1.2_{-0.8}^{+0.4} \mathrm{pc}$ is consistent with the interferometry results, although a little larger. This is understood because here we are considering dust within the torus that is colder than that traced by the MIR interferometry, as we include data at $\lambda>20$ $\mu \mathrm{m}$ in our fits. These colder clumps will be generally located at larger radii, which explain the value of $r_{\text {out }}$ obtained from the global posterior distribution. The torus outer radius including cooler dust would be larger still than the value of $r_{\text {out }} \sim 1.2 \mathrm{pc}$. Further studies including Herschel, SOFIA, and/or ALMA observations will help constrain the extent of the FIR-emitting dust. Indeed, some pilot studies already showed the importance of FIR data in tracing cooler dust (Ramos Almeida et al. 2011; García-Burillo et al. 2014).

\subsection{Distribution of Torus Model Parameters}

The key science direction of this paper is to investigate the torus morphology quantitatively by deriving torus parameters for each subgroup and comparing them. The median values of the model parameters fitted to our nuclear IR SEDs are reported in Table 4. The median values of the global posterior distributions of the free parameters $\sigma, Y, N_{0}, q$, and $\tau_{\mathrm{V}}$ for each subgroup are compiled in Table 5. As discussed in Section 4.2 , for the inclination angle $i$ we derive the median values from the individual galaxy fits for each subgroup.

Figure 2 shows the global posterior distributions of each physical parameter for the different subgroups. Black, blue, red, and green histograms show the parameter distribution of all, type-1, HBLR, and NHBLR sources, respectively.

As shown in Figure 2, for some of the parameters, the global distributions are similar for the three subgroups, but others are 

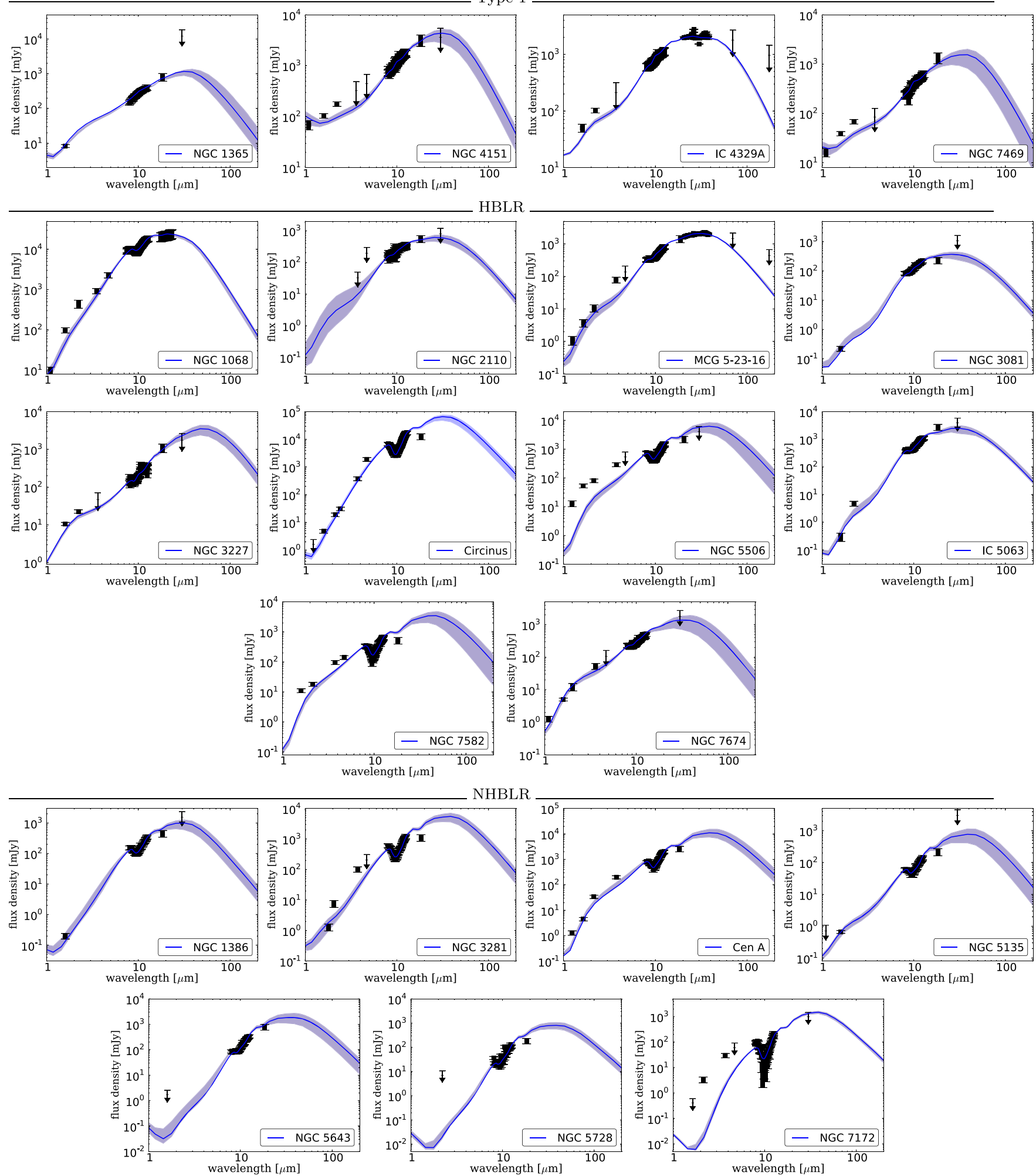

Figure 1. Clumpy torus model fits. The filled dots are the photometric data and the black line is the MIR spectrum. The upper limit points are shown as arrows. The solid blue lines are the models computed with the median value of the probability distribution of each parameter. The blue shaded areas indicate the range of models compatible with a $68 \%$ confidence interval. For the details on the calculation of the median values, see Section 3.

clearly different. In order to quantify these differences, we follow the same approach as in Ramos Almeida et al. (2011a). They used the Kullback-Leibler divergence (KLD; Kullback \& Leibler 1951) to show that the joint posterior distributions of type-1 and type-2 AGNs were quantitatively different. The
KLD takes into account the full shape of two posterior distributions to compare them. When the two distributions are identical, the value is $K L D=0$ and the larger the KLD value, the more different the two distributions. Ramos Almeida et al. (2011a) concluded that if KLD > 1.0, the two posteriors can be 
Table 4

Fitted Torus Model Parameters from SED + Spectroscopy Data

\begin{tabular}{|c|c|c|c|c|c|c|c|c|c|c|c|}
\hline$\overline{\text { Galaxy }}$ & $\begin{array}{l}\sigma_{\text {torus }} \\
{[\mathrm{deg}]}\end{array}$ & $Y$ & $N_{0}$ & $q$ & $\tau_{\mathrm{V}}$ & $\begin{array}{c}i \\
{[\mathrm{deg}]}\end{array}$ & $\overline{C C_{\mathrm{T}}}$ & $\begin{array}{c}\log L_{\text {bol }}^{(\bmod )} \\
{[\mathrm{erg} / \mathrm{s}]}\end{array}$ & $\begin{array}{c}r_{\text {in }} \\
{[\mathrm{pc}]}\end{array}$ & $\begin{array}{l}r_{\text {out }} \\
{[\mathrm{pc}]}\end{array}$ & $\begin{array}{c}H \\
{[\mathrm{pc}]}\end{array}$ \\
\hline \multicolumn{12}{|c|}{ Type-1 } \\
\hline NGC 4151 & $16_{-1}^{+1}$ & $19_{-4}^{+4}$ & $13_{-1}^{+1}$ & $1.6_{-0.3}^{+0.3}$ & $89_{-8}^{+8}$ & $71_{-2}^{+2}$ & $0.13_{-0.02}^{+0.03}$ & $43.9_{-0.1}^{+0.9}$ & $0.108_{-0.008}^{+0.007}$ & $2.1_{-0.5}^{+0.5}$ & $0.6_{-0.1}^{+0.2}$ \\
\hline IC $4329 A$ & $40_{-1}^{+1}$ & $8_{-1}^{+1}$ & $12_{-1}^{+1}$ & $0.5_{-0.1}^{+0.1}$ & $148_{-2}^{+1}$ & $4_{-3}^{+4}$ & $0.65_{-0.03}^{+0.03}$ & $44.4_{-0.1}^{+1.8}$ & $0.192_{-0.001}^{+0.002}$ & $1.7_{-0.0}^{+0.1}$ & $1.1_{-0.0}^{+0.0}$ \\
\hline NGC 7469 & $21_{-2}^{+2}$ & $22_{-4}^{+4}$ & $13_{-1}^{+1}$ & $1.3_{-0.3}^{+0.3}$ & $124_{-14}^{+12}$ & $59_{-4}^{+3}$ & $0.20_{-0.04}^{+0.05}$ & $44.6_{-0.1}^{+0.8}$ & $0.239_{-0.021}^{+0.020}$ & $5.3_{-1.2}^{+1.1}$ & $1.9_{-0.4}^{+0.4}$ \\
\hline NGC 1068 & $56_{-18}^{+8}$ & $6_{-1}^{+2}$ & $5_{-1}^{+3}$ & $0.6_{-0.4}^{+1.2}$ & $38_{-7}^{+3}$ & $67_{-5}^{+11}$ & $0.78_{-0.19}^{+0.06}$ & $44.4_{-0.1}^{+0.9}$ & $0.198_{-0.006}^{+0.015}$ & $1.3_{-0.1}^{+0.6}$ & $\overline{1.1_{-0.1}^{+0.3}}$ \\
\hline NGC 2110 & $55_{-8}^{+8}$ & $17_{-6}^{+7}$ & $9_{-2}^{+2}$ & $2.7_{-0.3}^{+0.2}$ & $146_{-4}^{+2}$ & $40_{-6}^{+5}$ & $0.87_{-0.11}^{+0.06}$ & $43.3_{-0.1}^{+1.0}$ & $0.058_{-0.002}^{+0.004}$ & $1.0_{-0.4}^{+0.5}$ & $0.8_{-0.3}^{+0.4}$ \\
\hline $\begin{array}{c}\text { MCG -5- } \\
23-16\end{array}$ & $58_{-8}^{+5}$ & $20_{-1}^{+2}$ & $7_{-1}^{+2}$ & $2.1_{-0.1}^{+0.2}$ & $144_{-6}^{+3}$ & $48_{-3}^{+7}$ & $0.85_{-0.05}^{+0.03}$ & $43.9_{-0.1}^{+1.2}$ & $0.114_{-0.003}^{+0.004}$ & $2.3_{-0.2}^{+0.3}$ & $2.0_{-0.3}^{+0.3}$ \\
\hline NGC 3081 & $62_{-5}^{+4}$ & $11_{-4}^{+9}$ & $12_{-1}^{+1}$ & $2.6_{-0.4}^{+0.2}$ & $98_{-11}^{+12}$ & $66_{-19}^{+13}$ & $0.96_{-0.04}^{+0.02}$ & $43.1_{-0.1}^{+0.9}$ & $0.043_{-0.003}^{+0.003}$ & $0.5_{-0.2}^{+0.5}$ & $0.4_{-0.2}^{+0.4}$ \\
\hline NGC 7582 & $53_{-2}^{+3}$ & $20_{-1}^{+2}$ & $12_{-2}^{+1}$ & $0.1_{-0.0}^{+0.1}$ & $79_{-9}^{+7}$ & $6_{-4}^{+5}$ & $0.90_{-0.02}^{+0.02}$ & $43.5_{-0.1}^{+0.9}$ & $0.070_{-0.004}^{+0.005}$ & $1.4_{-0.2}^{+0.3}$ & $1.2_{-0.2}^{+0.2}$ \\
\hline NGC 7674 & $39_{-9}^{+13}$ & $15_{-4}^{+5}$ & $8_{-3}^{+3}$ & $1.1_{-0.6}^{+0.5}$ & $133_{-15}^{+9}$ & $44_{-13}^{+13}$ & $0.56_{-0.20}^{+0.17}$ & $44.8_{-0.1}^{+0.5}$ & $0.330_{-0.038}^{+0.068}$ & $5.3_{-1.7}^{+2.5}$ & $3.3_{-1.0}^{+1.4}$ \\
\hline \multicolumn{12}{|c|}{ NHBLR } \\
\hline NGC 1386 & $56_{-9}^{+7}$ & $19_{-5}^{+5}$ & $8_{-1}^{+2}$ & $1.3_{-0.5}^{+0.3}$ & $37_{-4}^{+4}$ & $68_{-5}^{+9}$ & $0.87_{-0.10}^{+0.05}$ & $42.5_{-0.1}^{+0.8}$ & $0.023_{-0.001}^{+0.002}$ & $0.5_{-0.1}^{+0.2}$ & $0.4_{-0.1}^{+0.1}$ \\
\hline NGC 3281 & $68_{-2}^{+1}$ & $19_{-2}^{+3}$ & $14_{-1}^{+1}$ & $0.4_{-0.2}^{+0.2}$ & $38_{-4}^{+3}$ & $19_{-6}^{+6}$ & $0.99_{-0.01}^{+0.01}$ & $44.2_{-0.1}^{+0.9}$ & $0.151_{-0.008}^{+0.010}$ & $2.9_{-0.4}^{+0.6}$ & $2.7_{-0.4}^{+0.5}$ \\
\hline Cen A & $50_{-9}^{+10}$ & $17_{-3}^{+3}$ & $10_{-2}^{+2}$ & $0.3_{-0.2}^{+0.3}$ & $89_{-13}^{+11}$ & $38_{-9}^{+8}$ & $0.81_{-0.16}^{+0.10}$ & $42.5_{-0.1}^{+0.8}$ & $0.021_{-0.002}^{+0.002}$ & $0.4_{-0.1}^{+0.1}$ & $0.3_{-0.1}^{+0.1}$ \\
\hline NGC 5135 & $63_{-5}^{+3}$ & $17_{-2}^{+5}$ & $12_{-2}^{+1}$ & $0.4_{-0.3}^{+0.4}$ & $71_{-6}^{+5}$ & $17_{-10}^{+10}$ & $0.97_{-0.04}^{+0.01}$ & $43.6_{-0.1}^{+0.8}$ & $0.079_{-0.006}^{+0.007}$ & $1.4_{-0.3}^{+0.5}$ & $1.2_{-0.3}^{+0.4}$ \\
\hline NGC 5643 & $62_{-6}^{+4}$ & $14_{-2}^{+4}$ & $13_{-1}^{+1}$ & $0.8_{-0.5}^{+0.5}$ & $56_{-9}^{+11}$ & $74_{-12}^{+8}$ & $0.97_{-0.04}^{+0.02}$ & $43.0_{-0.1}^{+0.8}$ & $0.040_{-0.003}^{+0.004}$ & $0.6_{-0.1}^{+0.2}$ & $0.5_{-0.1}^{+0.2}$ \\
\hline NGC 5728 & $66_{-3}^{+2}$ & $17_{-1}^{+2}$ & $14_{-1}^{+1}$ & $0.7_{-0.4}^{+0.4}$ & $48_{-6}^{+7}$ & $80_{-8}^{+5}$ & $0.99_{-0.01}^{+0.01}$ & $43.4_{-0.1}^{+0.9}$ & $0.063_{-0.004}^{+0.004}$ & $1.1_{-0.1}^{+0.2}$ & $1.0_{-0.1}^{+0.2}$ \\
\hline
\end{tabular}

Note. Torus model parameters derived from the fits with BAYESClumpy. Median values of each posterior distribution are listed with their corresponding $\pm 1 \sigma$ values around the median.

Table 5

Torus Model Parameters from the Global Posterior Distributions

\begin{tabular}{|c|c|c|c|c|c|c|c|c|c|c|c|}
\hline $\begin{array}{l}\text { AGN } \\
\text { Type }\end{array}$ & $\begin{array}{l}\sigma_{\text {torus }} \\
{[\mathrm{deg}]} \\
\end{array}$ & $Y$ & $\overline{N_{0}}$ & $q$ & $\tau_{\mathrm{V}}$ & $\begin{array}{c}i \\
{[\mathrm{deg}]} \\
\end{array}$ & $\overline{C C_{\mathrm{T}}}$ & $\begin{array}{c}\log L_{\text {bol }}^{(\text {mod })} \\
{[\mathrm{erg} / \mathrm{s}]}\end{array}$ & $\begin{array}{c}r_{\text {in }} \\
{[\mathrm{pc}]}\end{array}$ & $\begin{array}{l}r_{\text {out }} \\
{[\mathrm{pc}]}\end{array}$ & $\begin{array}{c}H \\
{[\mathrm{pc}]}\end{array}$ \\
\hline All & $56_{-19}^{+6}$ & $18_{-6}^{+2}$ & $12_{-4}^{+1}$ & $0.6_{-0.5}^{+0.5}$ & $81_{-43}^{+23}$ & $48_{-37}^{+12}$ & $0.88_{-0.38}^{+0.06}$ & $43.4_{-0.5}^{+0.4}$ & $0.066_{-0.024}^{+0.048}$ & $1.2_{-0.8}^{+0.4}$ & $1.0_{-0.6}^{+0.3}$ \\
\hline HBLR & $56_{-8}^{+4}$ & $18_{-10}^{+1}$ & $10_{-3}^{+1}$ & $0.8_{-0.8}^{+1.1}$ & $98_{-57}^{+34}$ & $46_{-36}^{+14}$ & $0.88_{-0.14}^{+0.004}$ & $43.7_{-0.7}^{+0.0}$ & $0.072_{-0.030}^{+0.048}$ & $1.2_{-0.4}^{+0.8}$ & $1.0_{-0.3}^{+0.6}$ \\
\hline NHBLR & $64_{-11}^{+2}$ & $18_{-3}^{+2}$ & $13_{-3}^{+0}$ & $0.4_{-0.4}^{+0.2}$ & $43_{-11}^{+17}$ & $48_{-28}^{+17}$ & $0.96_{-0.10}^{+0.02}$ & $43.2_{-0.8}^{+0.1}$ & $0.060_{-0.042}^{+0.006}$ & $0.8_{-0.4}^{+0.8}$ & $1.0_{-0.6}^{+0.3}$ \\
\hline
\end{tabular}

Note. Torus parameters from the global posterior distributions of each subgroup.

considered significantly different. We calculate the KLD values for the global distributions of each torus parameter among the three groups. The values are reported in Table 6.

We find significant differences for the parameters $\sigma, Y$, and $N_{0}$ between type-1 AGNs and HBLR AGNs. The differences in these parameters between type-1 and type-2 AGNs were already reported in Ramos Almeida et al. (2011a) with larger significance, but based on fits to NIR and MIR photometry only, where spectroscopic data were not included. They also did not consider information from spectropolarimetry data, as we are doing here. Therefore, we confirm the results of Ramos Almeida et al. (2011a) after including $N$ band spectroscopy to the IR photometry, which is crucial to constrain the six torus parameters (Alonso-Herrero et al. 2011; Ramos Almeida et al. 2014). We also find that the parameters $\sigma, Y$, and $N_{0}$ of HBLR and NHBLR AGNs are significantly different. Considering the average values in Table 5, the tori of NHBLR AGNs have larger $\sigma$, larger $Y$, and larger $N_{0}$ than those of HBLR AGNs.

There are various possible interpretations for the difference in $\sigma$ among the subgroups. One possibility is that the smaller $\sigma$ could be due to larger AGN luminosities (receding torus model; Lawrence 1991; Ricci et al. 2013). However, as shown in Table 5, the differences in median bolometric AGN 

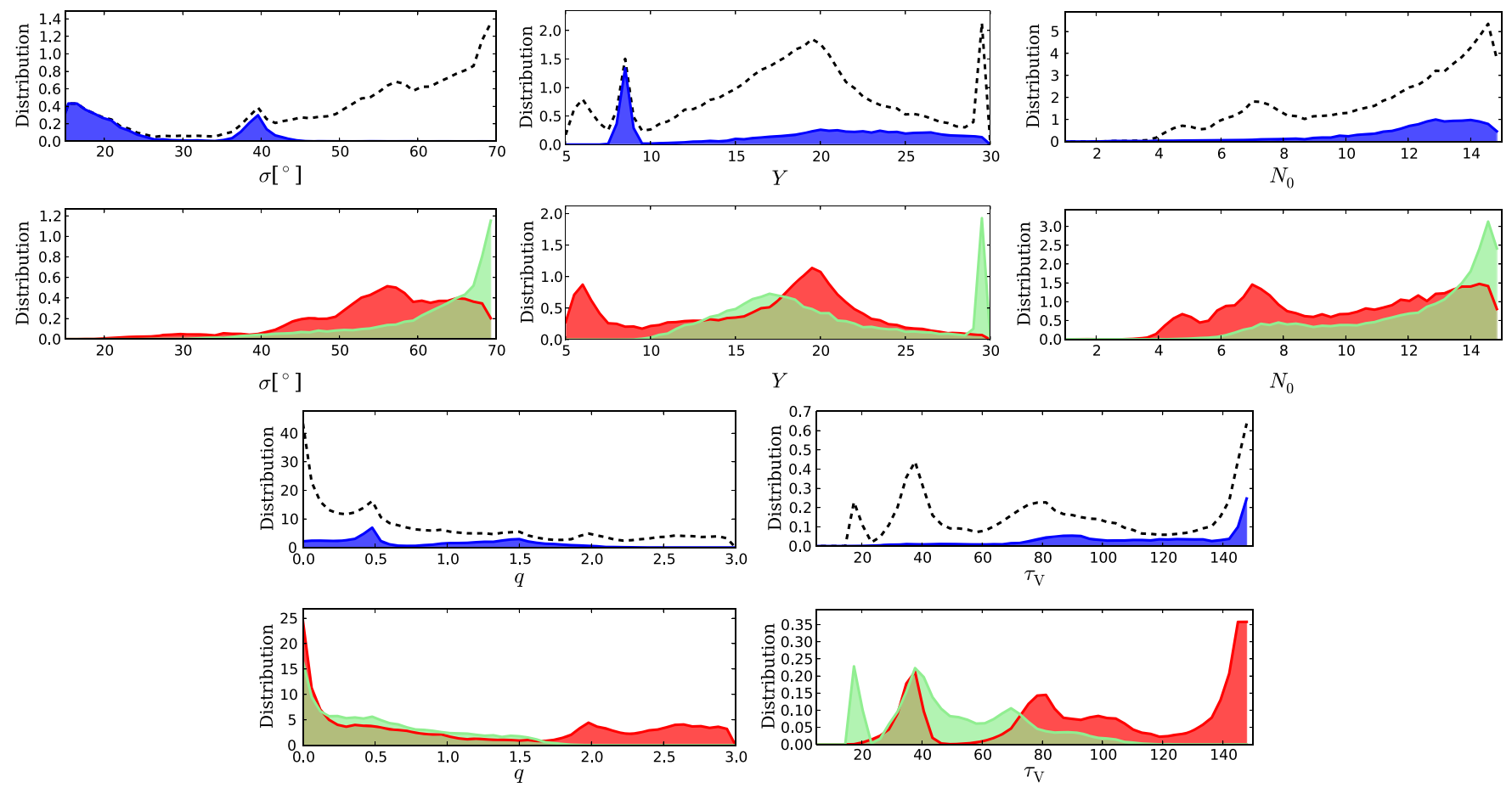

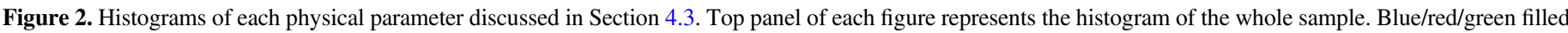
color represents the histogram of type-1/HBLR/NHBLR, respectively.

Table 6

Results of KLD Test for Each Parameter Among Each Subgroup

\begin{tabular}{lccccc}
\hline \hline AGN Type & $\sigma_{\text {torus }}$ & $Y$ & $N_{0}$ & $q$ & $\tau_{\mathrm{V}}$ \\
\hline Type-1 vs HBLR & $\mathbf{6 . 5 9}$ & $\mathbf{3 . 6 2}$ & $\mathbf{1 . 4 8}$ & 0.50 & 0.10 \\
Type-1 vs NHBLR & $\mathbf{4 . 0 2}$ & 0.45 & 0.94 & 0.30 & $\mathbf{8 . 5 0}$ \\
HBLR vs NHBLR & $\mathbf{2 . 4 5}$ & $\mathbf{4 . 1 1}$ & 0.21 & 0.83 & $\mathbf{1 . 7 3}$ \\
\hline
\end{tabular}

Note. KLD is calculated for the global posterior distribution of each parameter among the subgroups. Values larger than 1 are shown in bold.

luminosities among the subgroups are within the uncertainties. Therefore, we consider that the effect of the AGN luminosity is negligible in this study. Another possible interpretation is a selection bias in the optical type-1/type-2 AGN selection. Ramos Almeida et al. (2011a) and Elitzur (2012) discussed that AGN classification would depend on the distribution of the obscuring material; type-1 AGNs would be preferentially selected from lower-obscuration AGNs, while type-2 AGNs (HBLR and NHBLR) from higher-obscuration AGNs. This could be partly producing the differences in $\sigma$ that we found for type-1 and type-2 AGNs, but correcting this effect quantitatively is extremely difficult and beyond the scope of this paper.

\subsection{Distribution of Covering Factor}

As shown in Section 3.1, we can derive physical parameters of the torus model by combining the model parameters. The individual values of these physical parameters are reported in Table 4, and those obtained from the global posterior distribution for each subgroup are shown in Figure 3 and Table 5.

An interesting comparison can be made between the geometrical covering factor of the torus model $\left(C_{\mathrm{T}}\right.$; described in Equation (6)) of the different subgroups and the average column densities derived from X-ray data $\left(N_{\mathrm{H}}\right)$. NHBLRs have the largest column densities, with an average value of $\log N_{\mathrm{H}} \sim 24.0 \mathrm{~cm}^{-2} \quad$ (i.e., Compton-thick), followed by HBLRs, with $\log N_{\mathrm{H}} \sim 23.4 \mathrm{~cm}^{-2}$, and type-1s, with $\log N_{\mathrm{H}} \sim 21.8 \mathrm{~cm}^{-2}$. Based on hard X-ray $(50-200 \mathrm{keV})$ observations of nearby AGNs obtained with INTEGRAL, Ricci et al. (2011) reported differences between the X-ray reflection component of type-1 and type-2 AGNs. Type-1 and "lightly obscured" AGN with $N_{\mathrm{H}} \leqslant 10^{23} \mathrm{~cm}^{-2}$ have the same X-ray reflection component, with reflection amplitude $R \sim 0.4$. On the other hand, "mildly obscured" AGNs $\left(N_{\mathrm{H}} \geqslant 10^{23} \mathrm{~cm}^{-2}\right)$ show a clearly stronger X-ray reflection component with $R \sim 2.2$, suggesting that the central engine of "mildly obscured" AGNs would be covered by an X-ray reflection wall. Our results are in good agreement with Ricci et al. (2011) if we consider the $C_{\mathrm{T}}$ and $N_{\mathrm{H}}$ values for each subgroup. The type-1 AGNs in our sample fall under "lightly obscured" AGNs in their study, and indeed they show small covering factors $\left(C_{\mathrm{T}} \sim 0.18\right)$, suggesting a small torus $\mathrm{X}$-ray reflection solid angle. The HBLR and NHBLR AGN subgroups would fall in the "mildly obscured" AGN category, and we found large covering factors for them $\left(C_{\mathrm{T}} \sim 0.88\right.$ and 0.96 respectively), suggesting a larger X-ray reflection component (see also Ricci et al. 2014). Figure 4 shows a schematic illustration of the torus geometrical differences among type-1s (top), HBLRs (middle), and NHBLRs (bottom).

\subsection{Torus Model Morphological Differences Between HBLRs and NHBLRs}

In this section we focus on the differences between the modeled tori of HBLRs and NHBLRs. In the case of HBLRs, we obtain $\sigma$ values smaller than for NHBLRs, which is equivalent to larger torus opening angles $\left(90^{\circ}-\sigma\right)$. This implies that HBLR objects can have a larger scattering region. 

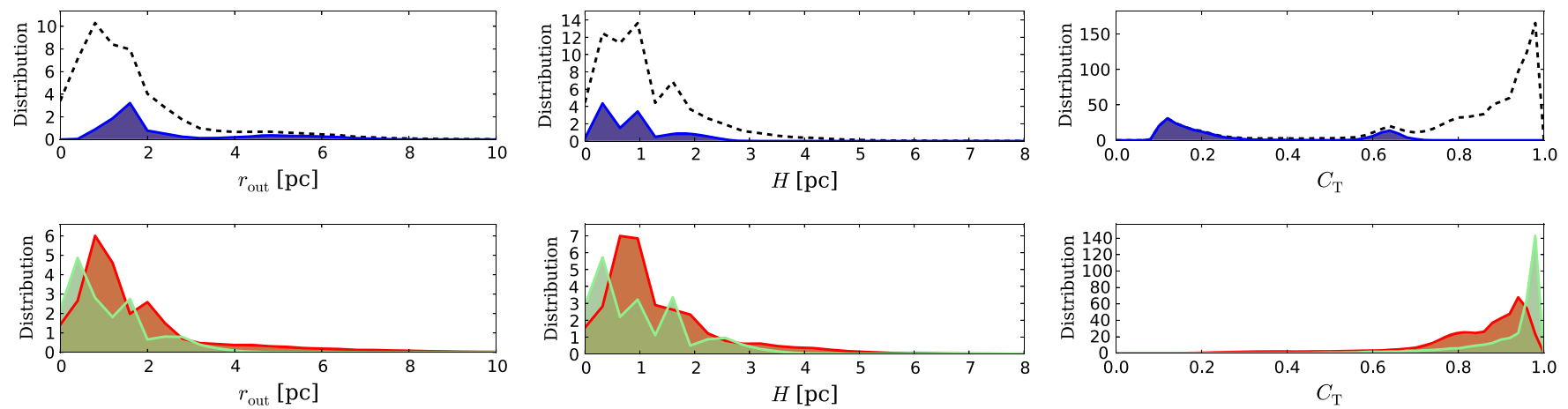

Figure 3. Same as in Figure 1, but for the $r_{\text {out }}, H$, and $C_{\mathrm{T}}$ parameters.
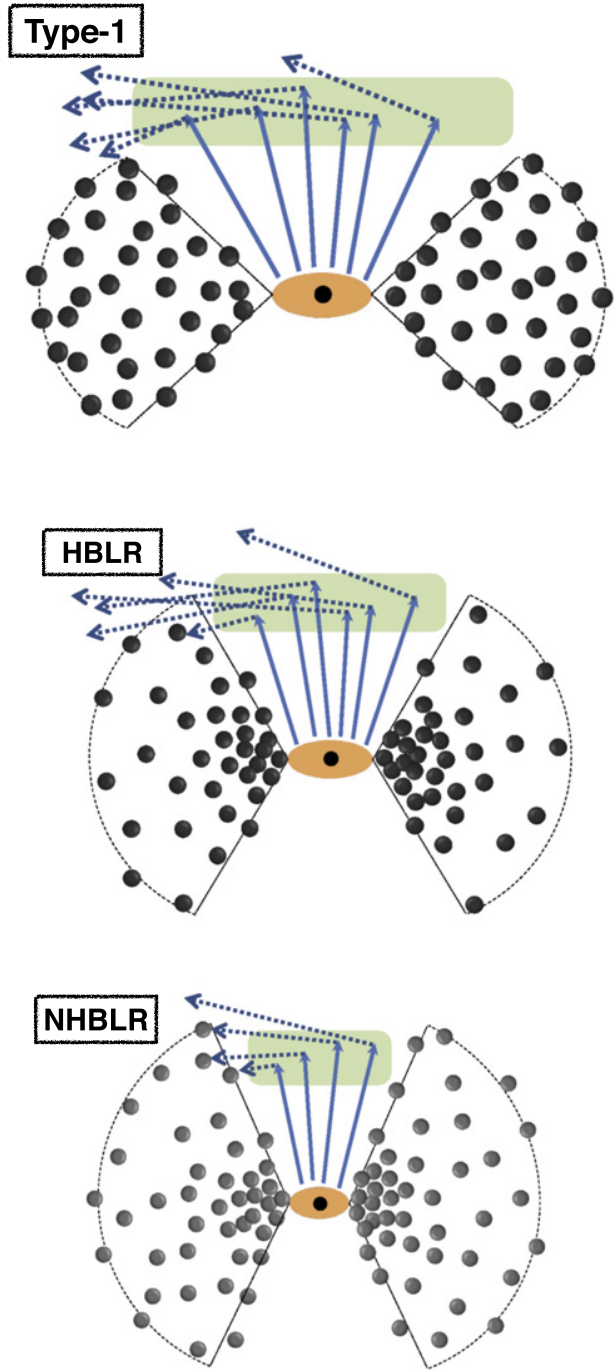

Figure 4. Schematic illustration of the torus geometry for type-1 AGNs (top), HBLRs (middle), and NHBLRs (bottom). The difference in color intensity between the two bottom panels shows the difference in optical depth of the clumps $\tau_{\mathrm{V}}$, where darker color means larger $\tau_{\mathrm{V}}$. The orange region represents the BLR. The green area represents the media where some of the incoming BLR emission is scattered and then polarized (in all diagrams we describe only scattering from the polar scattering region and ignore the inner equatorial scattering region, predominantly responsible for the polarized flux in Type 1 objects). The blue solid arrows represent the path of BLR photons and the blue dashed arrows represent the path of the polarized BLR photons. The observer is assumed to be on the left side of the torus with an inclination angle of $50^{\circ}, 46^{\circ}$, and $48^{\circ}$ (see Table 5). The only photons scattered along these lines of sight are shown. (see middle panel of Figure 4.) The scattering region (shown schematically as a filled green bar) can be larger due to the larger opening angle of the torus, allowing more photons to be scattered, and hence polarized, from the BLR. We note that the larger opening of the ionization cone, if unresolved, will produce a slightly lower degree of polarization due to (partial) cancellation of those polarization vectors at the edges of the scattering region. However, in our case, the increased amount of scattered photons will significantly increase the polarized flux, with only a very small reduction in the degree of polarization. To confirm this effect, we produced a toy polarization model assuming that the scattering region is a two-dimensional biconical structure centered on the central engine. Then, we measure the degree of polarization and polarized flux, and found that the measured polarized flux is larger for HBLR than those for NHBLR, supporting our results. Miller \& Goodrich (1990) also find the same results using a three-dimensional cone and more sophisticated modeling, and more complex assumptions.

In the case of the NHBLR, we obtain larger $\sigma$ values than for HBLR. This means that the probability that scattered radiation from the BLR can be blocked is higher than for HBLR objects. This is also in agreement with the larger value of $\log N_{\mathrm{H}} \sim 24.0 \mathrm{~cm}^{-2}$ estimated from X-ray observations of the NHBLR objects in our sample.

To summarize, as shown in the bottom panel of Figure 4, the chance to observe scattered (polarized) flux from the BLR is reduced by the double effect of (a) less scattering of the flux from the BLR (due to the reduced scattering area) and (b) more obscuration between the observer and the scattering region. Therefore, the classification of an AGN as either HBLR or NHBLR is probabilistic, and it would depend on the intrinsic properties of the torus, in particular of $\sigma$. This could be a reasonable explanation for the lack of a hidden (polarized) BLR in some type- 2 objects. ${ }^{17}$

However, we note that the classification of the galaxies as either HBLR or NHBLR is mainly based on spectropolarimetric observations from 3-4 $\mathrm{m}$ telescopes, and some of the NHBLRs could be then misclassified. Therefore, further higher sensitivity spectropolarimetry observations of NHBLR AGNs with $8 \mathrm{~m}$ class telescopes such as Subaru/FOCAS and/or VLT/ FORS2 are highly encouraged to search for the HBLR in those AGNs (C. Ramos Almeida et al. 2015, in preparation).

\footnotetext{
17 A similar explanation for the lack of HBLR detection in $\sim 40 \%$ of type-2 objects, based on the distribution of dust within the torus and its inclination being not as simple as predicted by the unified model, was shown in the talk by C. Ramos Almeida at the Polarization and Active Galactic Nuclei Workshop held on 2012 October 16-17 at the Royal Observatory of Belgium.
} 


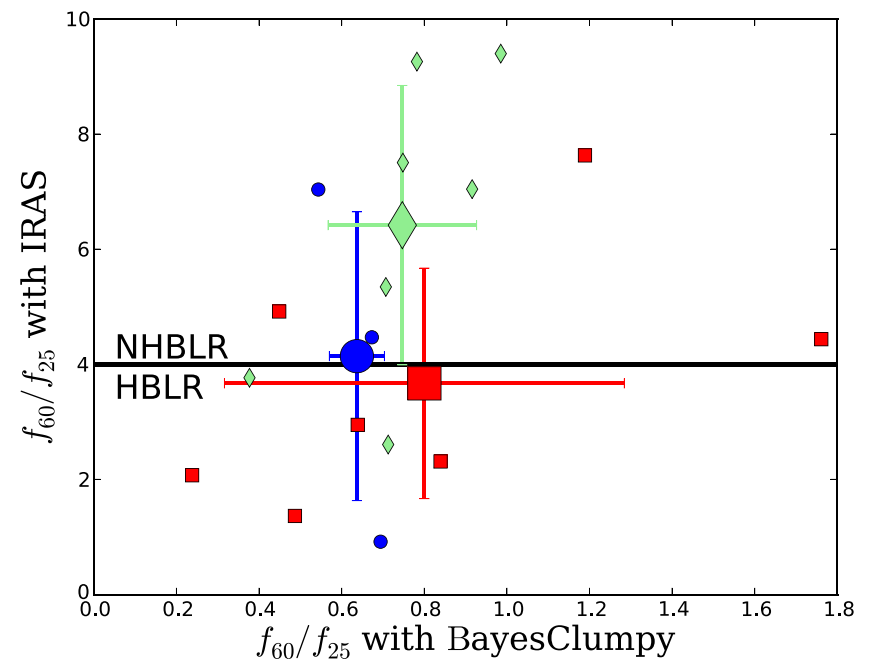

Figure 5. Plot comparing the $f_{60} / f_{25}$ colors measured from IRAS photometry and extrapolated from the clumpy torus model fits. Blue circle/red square/green diamond represents type-1, HBLR, and NHBLR AGN, respectively. The larger symbol represents the average value of each subgroup. The horizontal line at $f_{60} / f_{25}=4$ is the boundary between HBLR and NHBLR AGN according to Heisler et al. (1997), where HBLR AGN have $f_{60} / f_{25}<4$ and NHBLR AGN $f_{60} / f_{25}>4$.

\subsection{Inclination Angle Effect on Detectability of HBLR in Type-2 AGNs}

The dependence of HBLR detection in type-2 AGNs on torus inclination angle is still a matter of debate. This idea arises from the observational trend that the IRAS60 $\mu \mathrm{m}$ to $25 \mu \mathrm{m}$ color ratio $\left(f_{60} / f_{25}\right)$ of HBLR AGNs is $f_{60} / f_{25}<4$ on average while that of NHBLR AGNs is $f_{60} / f_{25}>4$ (Heisler et al. 1997; Lumsden et al. 2001). Several authors have suggested that this trend is due to the inclination angle of the torus: the cooler outer dust within the torus blocks the warm inner hot dust for edge-on views, producing the high $f_{60} / f_{25}$, while the warm inner dust can be seen from face-on views, reducing the value of $f_{60} / f_{25}$. Following this idea, type-2 AGNs with low $f_{60} / f_{25}$ would tend to be detected as HBLR AGNs due to the more face-on view of the torus and vice versa.

Here we can take advantage of the torus models fitted to our SEDs, which are available for each AGN and shown in Figure 1. The wavelength range covered by the models allows us to calculate the $f_{60} / f_{25}$ color ratios for each source. We have also compiled $f_{60} / f_{25}$ color ratios from IRAS for comparison, which probe much larger scales than our torus SEDs. Out of 21 sources, we obtained $17 \operatorname{IRAS} f_{60} / f_{25}$ colors with good quality of fluxes (FQUAL $=3$, which is the highest quality). ${ }^{18}$

Figure 5 shows the relationship between the $f_{60} / f_{25}$ flux ratios obtained from IRAS and those from the torus model SEDs. The averages $I R A S f_{60} / f_{25}$ flux ratio for type- 1 , HBLR, and NHBLR AGNs are $4.1 \pm 2.5,3.7 \pm 2.0$, and $6.4 \pm 2.4$, respectively, showing the previously mentioned correlation for HBLR and NHBLR AGNs, although with large error bars. However, when we compare the values obtained from the torus model SEDs, which exclude contamination from the host galaxy, we find that they are very similar for the three groups and smaller than the IRAS colors $\left(f_{60} / f_{25}=0.63 \pm 0.07, \quad 0.80 \pm 0.48, \quad\right.$ and

\footnotetext{
18 See Beichman et al. (1988), p. 1 for the definition of FQUAL in the IRAS catalogs. False detections may be included when FQUAL $<3$.
}

$0.75 \pm 0.18$ for type-1, HBLR, and NHBLR AGNs respectively).

These results show that the differences in the $\operatorname{IRAS} f_{60} / f_{25}$ among the three subgroups are not produced from the torus dust. A similar result was reported by Alexander (2001), but using X-ray observations. Although the standard deviations of the average values of the IRAS colors are large for the three subgroups, one possible explanation for the cooler IRAS colors of NHBLR in comparison with those of HBLR AGNs could be dust emission from stronger starbursts in their host galaxies. This is in good agreement with previous results showing that highly obscured AGNs tend to have higher star formation activity in their host galaxies (Goulding et al. 2012; Ichikawa et al. 2012a, 2012b, 2014a; Castro et al. 2014). Therefore, larger obscuration from the torus in NHBLR AGNs (as shown in Figure 4) and higher star formation activity in the host galaxy could be somehow coupled. For example, using threedimensional hydrodynamical simulations, Wada \& Norman (2002) showed that starbursts and supernovae within the central $100 \mathrm{pc}$ of host galaxies help in lifting up the torus, suggesting that high star formation activity could influence the scale height of the torus. Comparing the nuclear and overall star formation activity of AGNs with the torus obscuration is crucial to find out if they are coupled (e.g., Imanishi et al. 2011; Esquej et al. 2014; Ichikawa et al. 2014a).

\section{CONCLUSIONS}

We constructed 21 IR torus-dominated SEDs including high spatial resolution NIR and MIR photometry, MIR spectroscopy, and Spitzer and Herschel FIR fluxes. By performing SED fitting using clumpy torus models and a Bayesian approach we derived torus parameters such as the torus covering factor $\left(C_{\mathrm{T}}\right)$, the torus inner and outer radius $\left(r_{\text {in }}\right.$ and $\left.r_{\text {out }}\right)$, and the torus scale height $(H)$. We divided the sample into subgroups based on whether or not they are optically type1 , type-2 with observational hidden broad line region signs (HBLR), and type-2 without any observational broad line region signs (NHBLR). Our results are summarized as follows:

1. Under the assumption of a clumpy distribution of the dust, we obtained a quantitative description of the torus geometry and intrinsic properties. We found that the median torus outer radius for the whole sample $r_{\text {out }}=1.2$ $\mathrm{pc}$ is consistent with the results from MIR interferometry observations.

2. We found that the tori of Type-1 AGN have smaller $\sigma, Y$, $N_{\mathrm{H}}$, and $C_{\mathrm{T}}$ than those of HBLR and NHBLR. Moreover, the tori of NHBLR are thicker and therefore have higher $C_{\mathrm{T}}$ than those of HBLR. These differences in the torus properties of HBLR and NHBLR AGN would make it more difficult to detect hidden BLR in NHBLR.

3. Combining $f_{60} / f_{25}$ colors obtained from IRAS photometry and from torus model SEDs, we showed that the low $f_{60} /$ $f_{25}$ measured for HBLR using IRAS data are not due to a more face-on inclination of the torus, but rather to star formation activity in their host galaxies.

We are grateful for useful comments from the anonymous referee. We also thank C. Ricci, T. Kawamuro, K. Matsuoka, and M. Stalevski for useful comments and discussions. K.I. thanks the Department of Physics and Astronomy at University of Texas at San Antonio, where most of the research was 
conducted. This paper is based on observations obtained at the Gemini Observatory, which is operated by the Association of Universities for Research in Astronomy, Inc., under a cooperative agreement with the NSF on behalf of the Gemini partnership. This work was partly supported by the Grant-inAid for JSPS Fellows for young researchers (K.I.). C.P. acknowledges support from UTSA and NSF (grant number 0904421). C.R.A. is supported by a Marie Curie Intra European Fellowship within the 7th European Community Framework Programme (PIEF-GA-2012-327934). A.A.-H. acknowledges support from the Spanish Plan Nacional de Astronomia y Astrofisica under grant AYA2012-31447.

\section{REFERENCES}

Alexander, D. M., Hough, J. H., Young, S., et al. 1999, MNRAS, 303, L17 Alexander, D. M. 2001, MNRAS, 320, L15

Alonso-Herrero, A., Quillen, A. C., Rieke, G. H., Ivanov, V. D., \& Efstathiou, A. 2003, AJ, 126, 81

Alonso-Herrero, A., Ramos Almeida, C., Mason, R., et al. 2011, ApJ, 736, 82 Alonso-Herrero, A., Sánchez-Portal, M., Ramos Almeida, C., et al. 2012a, MNRAS, 425, 311

Alonso-Herrero, A., Pereira-Santaella, M., Rieke, G. H., \& Rigopoulou, D. 2012b, ApJ, 744, 2

Antonucci, R. R. J., \& Miller, J. S. 1985, ApJ, 297, 621

Antonucci, R. 1993, ARA\&A, 31, 473

Asensio Ramos, A., \& Ramos Almeida, C. 2009, ApJ, 696, 2075

Asmus, D., Gandhi, P., Smette, A., Hönig, S. F., \& Duschl, W. J. 2011, A\&A, 536, A36

Beichman, C. A., Neugebauer, G., Habing, H. J., Clegg, P. E., \& Chester, T. J. 1988, Infrared Astronomical Satellite (IRAS) Catalogs and Atlases, Vol. 1: Explanatory Supplement (NASA RP-1190; Washington, DC: GPO)

Blanco, P. R., Ward, M. J., \& Wright, G. S. 1990, MNRAS, 242, 4P

Brightman, M., \& Nandra, K. 2011, MNRAS, 413, 1206

Brewer, B. J., \& Elliott, T. M. 2014, MNRAS, 439, L31

Carollo, C. M., Stiavelli, M., Seigar, M., de Zeeuw, P. T., \& Dejonghe, H. 2002, AJ, 123, 159

Castro, A., Miyaji, T., Shirahata, M., et al. 2014, PASJ, 66, 110

Deo, R. P., Richards, G. T., Crenshaw, D. M., \& Kraemer, S. B. 2009, ApJ, 705, 14

Efstathiou, A., \& Rowan-Robinson, M. 1995, MNRAS, 273, 649

Elitzur, M. 2012, ApJL, 747, L33

Elitzur, M., Ho, L. C., \& Trump, J. R. 2014, MNRAS, 438, 3340

Elvis, M., Wilkes, B. J., McDowell, J. C., et al. 1994, ApJS, 95, 1

Esquej, P., González-Martín, A., Gonzalez-Martin, O., et al. 2014, ApJ, 780,86

Feltre, A., Hatziminaoglou, E., Fritz, J., \& Franceschini, A. 2012, MNRAS, 426, 120

Galliano, E., \& Alloin, D. 2008, A\&A, 487, 519

Gandhi, P., Horst, H., Smette, A., et al. 2009, A\&A, 502, 457

García-Burillo, S., Combes, F., Usero, A., et al. 2014, A\&A, 567, A125

González-Martín, O., Rodríguez-Espinosa, J. M., Díaz-Santos, T., et al. 2013, A\&A, 553, A35

Goulding, A. D., Alexander, D. M., Bauer, F. E., et al. 2012, ApJ, 755, 5

Gu, Q., Maiolino, R., \& Dultzin-Hacyan, D. 2001, A\&A, 366, 765

Guainazzi, M., Rodriguez-Pascual, P., Fabian, A. C., Iwasawa, K., \& Matt, G. 2004, MNRAS, 355, 297

Heisler, C. A., Lumsden, S. L., \& Bailey, J. A. 1997, Natur, 385, 700

Ho, L. C., Filippenko, A. V., \& Sargent, W. L. W. 1997, ApJS, 112, 315

Hönig, S. F., Beckert, T., Ohnaka, K., \& Weigelt, G. 2006, A\&A, 452, 459

Hönig, S. F., \& Kishimoto, M. 2010, A\&A, 523, A27

Hönig, S. F., Leipski, C., Antonucci, R., \& Haas, M. 2011, ApJ, 736, 26

Hönig, S. F., Gandhi, P., Asmus, D., et al. 2014, MNRAS, 438, 647

Ichikawa, K., Ueda, Y., Terashima, Y., et al. 2012a, ApJ, 754, 45

Ichikawa, K., Ueda, Y., Terashima, Y., et al. 2012b, Torus Workshop, 2012, 109

Ichikawa, K., Imanishi, M., Ueda, Y., et al. 2014a, ApJ, 794, 139

Imanishi, M. 2002, ApJ, 569, 44

Imanishi, M., Ichikawa, K., Takeuchi, T., et al. 2011, PASJ, 63, 447
Itoh, T., Done, C., Makishima, K., et al. 2008, PASJ, 60, 251

Jiménez-Bailón, E., Santos-Lleó, M., Dahlem, M., et al. 2005, A\&A, 442, 861

Kawaguchi, T., \& Mori, M. 2011, ApJ, 737, 105

Kishimoto, M., Hönig, S. F., Beckert, T., \& Weigelt, G. 2007, A\&A, 476, 713

Kishimoto, M., Hönig, S. F., Antonucci, R., et al. 2009, A\&A, 507, L57

Kishimoto, M., Hönig, S. F., Antonucci, R., et al. 2011, A\&A, 536, 78

Koshida, S., Yoshii, Y., Kobayashi, Y., et al. 2009, ApJL, 700, L109

Kullback, S., \& Leibler, A. 1951, Ann. Math. Stat, 22, 79

Lawrence, A. 1991, MNRAS, 252, 586

Levenson, N. A., Heckman, T. M., Krolik, J. H., Weaver, K. A., \& Zdotycki, P. T. 2006, ApJ, 648, 111

Lira, P., Videla, L., Wu, Y., et al. 2013, ApJ, 764, 159

Lumsden, S. L., Heisler, C. A., Bailey, J. A., Hough, J. H., \& Young, S. 2001, MNRAS, 327, 459

Lumsden, S. L., Alexander, D. M., \& Hough, J. H. 2004, MNRAS, 348, 1451

Maiolino, R., \& Rieke, G. H. 1995, ApJ, 454, 95

Marin, F. 2014, MNRAS, 441, 551

Marinucci, A., Bianchi, S., Nicastro, F., Matt, G., \& Goulding, A. D. 2012 ApJ, 748, 130

Meisenheimer, K., Tristram, K. R. W., Jaffe, W., et al. 2007, A\&A, 471, 453

Meléndez, M., Mushotzky, R. F., Shimizu, T. T., Barger, A. J., \& Cowie, L. L. 2014, ApJ, 794, 152

Miller, J. S., \& Goodrich, R. W. 1990, ApJ, 355, 456

Moran, E. C., Barth, A. J., Kay, L. E., \& Filippenko, A. V. 2000, ApJL, 540, L73

Moran, E. C., Kay, L. E., Davis, M., Filippenko, A. V., \& Barth, A. J. 2001 ApJL, 556, L75

Moran, E. C., Filippenko, A. V., \& Chornock, R. 2002, ApJL, 579, L71

Nagar, N. M., Oliva, E., Marconi, A., \& Maiolino, R. 2002, A\&A, 391, L21

Nenkova, M., Ivezić, Ž, \& Elitzur, M. 2002, ApJL, 570, L9

Nenkova, M., Sirocky, M. M., Ivezić, Ž., \& Elitzur, M. 2008a, ApJ, 685, 147

Nenkova, M., Sirocky, M. M., Nikutta, R., Ivezić, Ž., \& Elitzur, M. 2008b, ApJ, 685, 160

Netzer, H., Lira, P., Trakhtenbrot, B., Shemmer, O., \& Cury, I. 2007, ApJ, 671,1256

Nicastro, F., Martocchia, A., \& Matt, G. 2003, ApJL, 589, L13

Nikutta, R., Elitzur, M., \& Lacy, M. 2009, ApJ, 707, 1550

Ossenkopf, V., Henning, T., \& Mathis, J. S. 1992, A\&A, 261, 567

Peng, Z., Gu, Q., Melnick, J., \& Zhao, Y. 2006, A\&A, 453, 863

Pier, E. A., \& Krolik, J. H. 1992, ApJ, 401, 99

Pier, E. A., \& Krolik, J. H. 1993, ApJ, 418, 673

Polletta, M., Weedman, D., Hönig, S., et al. 2008, ApJ, 675, 960

Quillen, A. C., McDonald, C., Alonso-Herrero, A., et al. 2001, ApJ, 547, 129

Ramos Almeida, C., Pérez García, A. M., Acosta-Pulido, J. A., \& González-Martín, O. 2008, ApJL, 680, L17

Ramos Almeida, C., Levenson, N. A., Rodr $\imath^{\prime}$ guez Espinosa, J. M., et al. 2009, ApJ, 702, 1127

Ramos Almeida, C., Levenson, N. A., Alonso-Herrero, A., et al. 2011a, ApJ, 731, 92

Ramos Almeida, C., Sánchez-Portal, M., Pérez García, A. M., et al. 2011, MNRAS, 417, L46

Ramos Almeida, C., Alonso-Herrero, A., Levenson, N. A., et al. 2014, MNRAS, 439, 3847

Reunanen, J., Kotilainen, J. K., \& Prieto, M. A. 2003, MNRAS, 343, 192

Ricci, C., Walter, R., Courvoisier, T. J.-L., \& Paltani, S. 2011, A\&A, 532, A102

Ricci, C., Paltani, S., Awaki, H., et al. 2013, A\&A, 553, A29

Ricci, C., Ueda, Y., Ichikawa, K., et al. 2014, A\&A, 567, A142

Scarrott, S. M., Draper, P. W., Stockdale, D. P., \& Wolstencroft, R. D. 1993, MNRAS, 264, L7

Schartmann, M., Meisenheimer, K., Camenzind, M., et al. 2008, A\&A, 482, 67

Shu, X. W., Wang, J. X., Jiang, P., Fan, L. L., \& Wang, T. G. 2007, ApJ, 657, 167

Simpson, C. 1998, ApJ, 509, 653

Stalevski, M., Fritz, J., Baes, M., Nakos, T., \& Popovic, L. 2012, MNRAS, 240, 2756

Tran, H. D. 2001, ApJL, 554, L19

Tran, H. D. 2003, ApJ, 583, 632

Tran, H. D., Lyke, J. E., \& Mader, J. A. 2011, ApJL, 726, L21

Tueller, J., Mushotzky, R. F., Barthelmy, S., et al. 2008, ApJ, 681, 113

Véron-Cetty, M.-P., \& Véron, P. 2006, A\&A, 455, 773

Wada, K., \& Norman, C. A. 2002, ApJL, 566, L21

Wang, J.-M., \& Zhang, E.-P. 2007, ApJ, 660, 1072 